\title{
Studying biological tissue with fluorescence lifetime imaging: microscopy, endoscopy, and complex decay profiles
}

\author{
Jan Siegel, Daniel S. Elson, Stephen E. D. Webb, K. C. Benny Lee, Alexis Vlandas, \\ Giovanni L. Gambaruto, Sandrine Lévêque-Fort, M. John Lever, Paul J. Tadrous, \\ Gordon W. H. Stamp, Andrew L. Wallace, Ann Sandison, Tim F. Watson, \\ Fernando Alvarez, and Paul M. W. French
}

\begin{abstract}
We have applied fluorescence lifetime imaging (FLIM) to the autofluorescence of different kinds of biological tissue in vitro, including animal tissue sections and knee joints as well as human teeth, obtaining two-dimensional maps with functional contrast. We find that fluorescence decay profiles of biological tissue are well described by the stretched exponential function (StrEF), which can represent the complex nature of tissue. The StrEF yields a continuous distribution of fluorescence lifetimes, which can be extracted with an inverse Laplace transformation, and additional information is provided by the width of the distribution. Our experimental results from FLIM microscopy in combination with the StrEF analysis indicate that this technique is ready for clinical deployment, including portability that is through the use of a compact picosecond diode laser as the excitation source. The results obtained with our FLIM endoscope successfully demonstrated the viability of this modality, though they need further optimization. We expect a custom-designed endoscope with optimized illumination and detection efficiencies to provide significantly improved performance. (C) 2003 Optical Society of America

OCIS codes: $170.2520,170.6920,170.3880$.
\end{abstract}

\section{Introduction}

The field of fluorescence imaging for biomedical applications has undergone a dramatic expansion over

When this research was performed, J. Siegel, D. S. Elson, S. E. D. Webb, K. C. Benny Lee, A. Vlandas, G. L. Gambaruto, S. LévêqueFort, M. J. Lever, P. J. Tadrous, G. W. H. Stamp, A. L. Wallace, A. Sandison, and P. M. W. French (paul.french@ic.ac.uk) were with the Photonics Group, Department of Physics, Imperial College of Science, Technology and Medicine, Prince Consort Road, London SW7 2BW, UK. F. Alvarez was with Departamento de Física de Materiales, Facultad de Química, Paseo Manuel de Lardizabal, 3, 20018 San Sebastián, Spain. J. Siegel is now with the Instituto de Optica, CSIC, C/Serrano 121, 28006 Madrid, Spain. S. Lévêque-Fort is now with Laboratoire de Photophysique Moleculaire, 91405 Orsay, France. M. J. Lever is with Department of Bioengineering. P. J. Tadrous and G. W. H. Stamp are with the Department of Histopathology, Hammersmith Hospital Campus. A. L. Wallace and A. Sandison are with the Department of Musculoskeletal Surgery, Charing Cross Hospital Campus. T. F. Watson is with the Department of Conservative Dentistry, Guys, Kings and St. Thomas' Dental Institute, London, SE1 9RT, UK.

Received 30 August 2002; revised manuscript received 23 October 2002 .

0003-6935/03/162995-10\$15.00/0

(C) 2003 Optical Society of America the past ten years. In contrast to reflected or transmitted light microscopy, which mainly images tissue structures, fluorescence microscopy can provide information about tissue composition and can indicate the state of individual tissue constituents through analysis of the fluorescence properties. This technique can derive intrinsic contrast from autofluorescence emitted from endogenous tissue fluorophores (such as elastin, collagen, tryptophan, Nicotinamide adenine dinucleotide, porphyrins, flavins, and the like), which have absorption bands in the near ultraviolet or blue and characteristic spectra in the visible region. ${ }^{1}$ Exogenous fluorescent markers with a high quantum yield can also be used to achieve a stronger extrinsic fluorescence contrast. In this case information is obtained from the change of the fluorescence properties of the marker during interaction with the different tissue components, or by selective localization of the marker in certain tissue constituents. ${ }^{2}$ Such fluorophores allow disease demarcation by fluorescence imaging and, in some cases, can also be exploited for photodynamic therapy. ${ }^{3}$ However, unwanted influences of extrinsic fluorophores on the processes studied and considerations of toxicity make autofluorescence microscopy more desirable. 
Fluorescence imaging usually entails acquiring a map of steady-state fluorescence intensity in a particular spectral emission band and achieves optical contrast between healthy and diseased tissues through their different emission intensities. The drawback of steady-state intensity-based techniques is their dependence on fluorophore concentration, which often exhibits local variations, on attenuation by reabsorption and/or scattering of the fluorescence in certain tissue structures and on local variations in the quantum yield (quenching) that result from the local fluorophore environment. Although some of these effects can be accounted for with "wavelengthratiometric" techniques at different excitation and/or emission wavelengths, this approach depends on the fortuitous availability of appropriate fluorescence properties or relies on the use of suitable extrinsic markers to provide contrast. ${ }^{4,5}$

A powerful alternative approach that is almost insensitive to local intensity variations or fluorophore concentration is fluorescence lifetime imaging (FLIM), which is based on the simultaneous measurement of the temporal decay in fluorescence intensity following laser excitation at every spatial point in a sample. Because the fluorescence lifetime is derived from relative intensity values, it can provide useful information concerning biological tissue in spite of the heterogeneity and strong optical scattering. FLIM thus provides a powerful functional imaging modality for biomedicine, providing maps of the spatial distribution of fluorescence lifetimes. FLIM has already been applied to studies of tissue constituents, ${ }^{6}$ cell cultures, ${ }^{7}$ and the human skin. ${ }^{8,9}$ FLIM can be performed either in the frequency domain, for which a high-frequency modulated laser beam excites the sample and the fluorescence lifetime is determined from the demodulation and phase shift of the fluorescence signal, ${ }^{10}$ or in the time domain, for which the fluorescence decay is directly measured after pulsed laser excitation. ${ }^{11}$ With either approach, functional information may be derived from the fluorescence lifetime $\tau$ by means of its dependence on the radiative decay rate $k_{r}$ and the nonradiative decay rate $k_{n r}$ of the fluorophore, where $\tau=1 /\left(k_{r}+\right.$ $k_{n r}$ ). As a consequence, the fluorescence lifetime is not only sensitive to the type of fluorophore but also depends on its environment. This functionality has been exploited to quantify physiological parameters including $\mathrm{pH},\left[\mathrm{Ca}^{2+}\right]$ and $\mathrm{pO}_{2}$ (for an overview, see Ref. 12 and references therein). Because of variations in the local environment and the wide range of protein conformations often present in biological tissue, the observed fluorescence decay profiles are often complex. Conventionally, such profiles have been analyzed with a multiexponential model, but we show that the stretched exponential function (StrEF) is more appropriate and provides lifetime images with superior signal-to-noise $(\mathrm{S} / \mathrm{N})$ ratios. ${ }^{13}$

An important aspect of any biomedical imaging technique is its viability for in vivo studies, in particular its compatibility with endoscopy, which facilitates noninvasive or minimally invasive optical biopsy. To date there have been relatively few reports of fluorescence endoscopy. White-light endoscopy often has a low diagnostic sensitivity and specificity, owing to intensity artifacts arising from specular reflection. ${ }^{14}$ Crossed-polarizer detection eliminates this problem, detecting only diffuse reflections, and can also correct a simultaneously acquired fluorescence image with nonhomogeneous illumination. ${ }^{15}$ Using this technique, $\mathrm{Qu}^{16}$ recently reported a real-time fluorescence/reflectance-ratio imaging endoscope capable of locating early malignant lesions in vivo. The combined use of endoscopy and multispectral imaging can provide additional contrast to identify tissue by means of its characteristic autofluorescence signature. ${ }^{17}$ The use of extrinsic photosensitizers, preferentially accumulated in tumors, was successfully demonstrated by McKechnie et al. using a custom-designed fluorescence endoscope for clinical studies of gastrointestinal cancers. ${ }^{18}$ These endoscopes have all relied on wide-field imaging. Several types of scanning confocal endomicroscope have also been reported, for example. ${ }^{19,20}$ We note, however, that the application of FLIM to endoscopy is limited to one report, ${ }^{21}$ which used the frequency-domain approach.

In this paper we report our application of FLIM to the study of biological tissue and our progress toward a time-domain FLIM endoscope. After a brief review of appropriate decay models for fluorescence lifetime data from real tissues, we present in vitro microscopy studies of the complex fluorescence decay profiles observed in biological tissue, obtaining fluorescence lifetime maps that demonstrate functional contrast between different types or states of tissue. We demonstrate that the stretched exponential function, as opposed to a single-exponential or multiexponential function, is the most appropriate model to fit these complex fluorescence decays and has the added benefit of providing an extra contrast parameter related to the width of the fluorescence lifetime distribution. The issue of portability and low cost of a clinically deployable FLIM system is addressed by the demonstration of a compact pulsed diode laser as the excitation source in this FLIM microscope. First results obtained from our time-domain endoscopic FLIM system are then presented, together with a discussion of their limitations.

\section{Appropriate Decay Models for Autofluorescence of Biological Tissue}

Although the fluorescence lifetime $\tau$ refers to the $a v$ erage time the molecule remains in its excited state, the actual decay dynamics are often complex. The simplest description for the decay of the fluorescence intensity $I(t)$ from its initial value $I_{0}$ is given by a single exponential decay yielding a single fluorescence lifetime $\tau_{0}$ :

$$
I(t)=I_{0} \exp \left(-\frac{t}{\tau_{0}}\right)+\text { const. }
$$


This model applies only to a very limited number of fluorescent molecules. A more general approach is to fit several decay constants, $\tau_{i}$, to a fluorescence decay profile, although such a multiexponential model greatly increases the calculation time, which becomes a severe problem for two-dimensional imaging:

$$
\begin{aligned}
I(t) & =\sum_{i}^{n} I_{i} \exp \left(-\frac{t}{\tau_{i}}\right)+\text { const. } \\
n & =1,2,3, \ldots
\end{aligned}
$$

For instance, the autofluorescence decays of collagen, elastin, and other tissue components measured by FLIM do not fit a single exponential decay profile, and a double exponential decay model provides a better fit. ${ }^{6}$ However, an apparently satisfactory fit to a model with discrete lifetimes can conceal the actual complexity in the decay mechanisms. ${ }^{22}$ For instance, interaction between fluorophores in heterogeneous samples can yield complex decay mechanisms that may result in a continuous distribution of fluorescence lifetimes. ${ }^{23}$ There are also many other situations in which one does not expect a limited number of discrete decay times. For instance, a single-tryptophan protein can have a large number of different conformations, whose distribution may lead to a continuous distribution of fluorescence lifetimes. ${ }^{23,24}$ Another possibility is a protein that has so many tryptophan residues that it is not practical to consider the individual decay times. ${ }^{12}$ Generally, these variations are present on a molecular scale and therefore cannot be spatially resolved with conventional optical techniques and so have to be accounted for in the decay model.

For these reasons, an erroneous assumption of a number of discrete lifetime components may lead to image artifacts when FLIM maps are fitted to multiexponential models. We have proposed an alternative approach to fit complex fluorescence decay images of biological tissue, employing the stretched exponential function (StrEF), also known as the Kohlrausch-Williams-Watts function,

$$
I(t)=I_{0} \exp \left[-\left(\frac{t}{\tau_{k w w}}\right)^{1 / h}\right]+\text { const. }
$$

where $\tau_{k w w}$ is the decay constant and $h$ the heterogeneity parameter. ${ }^{13}$ Our motivation to apply the StrEF to FLIM was triggered by the mathematical observation that the stretched exponential decay can be expressed as a continuous distribution of lifetimes,

$$
I(t)=\int_{0}^{\infty} \exp \left(-\frac{t}{\tau}\right) \rho(\tau) \mathrm{d} \tau .
$$

For correctly describing the decay given by Eq. (3) it is necessary to interpret the lifetimes in a statistical manner, which can be done by use of the mean lifetime of the distribution, ${ }^{25}$ given by

$$
\langle\tau\rangle=h \tau_{k w w} \Gamma[h],
$$

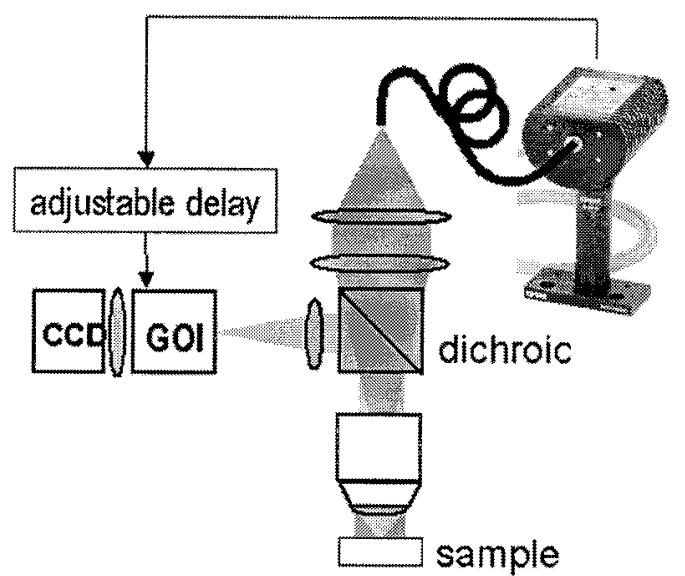

Fig. 1. Experimental setup for a compact FLIM microscopy system. The picosecond diode laser is fiber coupled, and its electronic trigger output is used to synchronize the gated optical intensifier (GOI) through an adjustable delay.

where $\Gamma[h]$ is the gamma function. Arbitrary choices of the number of exponential terms to represent complex fluorescence decay profiles are thus no longer necessary, and fitting to a stretched exponential can significantly decrease the required processing time compared with a multiexponential model, owing to the smaller number of fitting parameters (three for single exponential, four for StrEF, five for double exponential, seven for triple exponential). In our recent research we have demonstrated that this model describes our experimental time-domain widefield FLIM data of tissue samples significantly better than single-exponential or multiexponential decay models, yielding superior contrast in tissue discrimination. ${ }^{13}$ In this study we apply the StrEF to new FLIM data and provide analytical evidence of the appropriateness of this function by extracting the exponential decay components that are present in our experimental data.

\section{Fluorescence Lifetime Imaging Microscopy}

Our FLIM microscope operates in the time domain, and data are obtained by means of acquiring a series of time-gated fluorescence intensity images at increasing delays after excitation by ultrashort laser pulses, as described in detail in Ref. 26. Briefly, a home-built fluorescence microscope is extended to perform FLIM measurements, making use of a pulsed laser source for excitation and a gated optical intensifier (GOI) to generate wide-field time-gated fluorescence images of the sample (Fig. 1). The laser source used for the FLIM microscopy results shown in this study was a compact picosecond diode laser emitting optical pulses with a width of less than $35 \mathrm{ps}$ at a wavelength of $405 \mathrm{~nm}$ operating at a repetition rate of $40 \mathrm{MHz}$ with an average power of $0.5 \mathrm{~mW}$. These specifications make the diode laser a suitably compact and inexpensive light source for timedomain FLIM microscopy. The GOI has a gate width that is variable between $200 \mathrm{ps}$ and $1 \mathrm{~ns}$ and the gain applied to the double microchannel plate for 


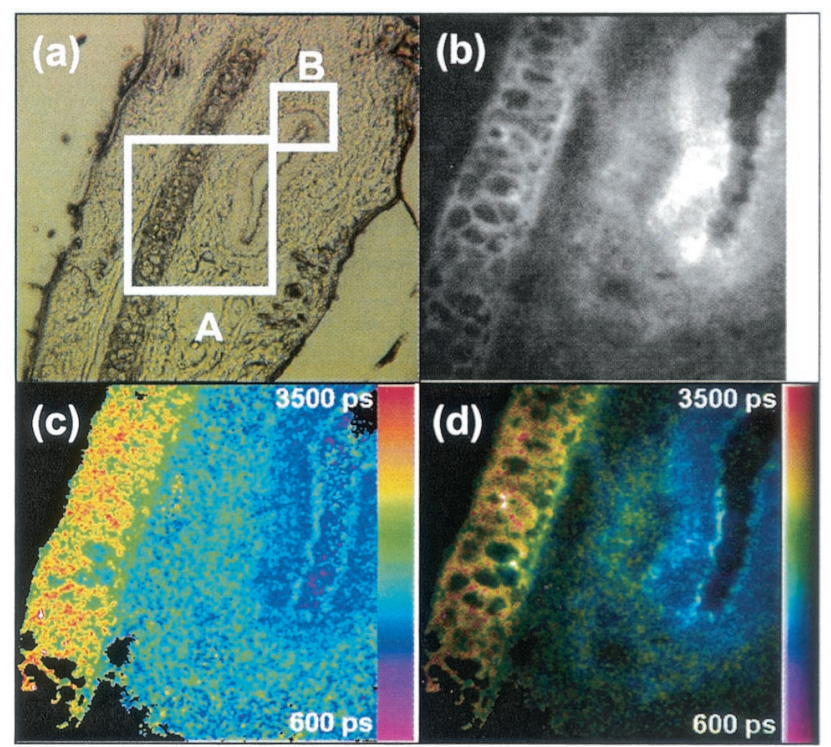

Fig. 2. (a) Light microscopy image of a $15-\mu \mathrm{m}$-thick unstained section of rat tissue. The two marked regions indicate the locations where FLIM microscopy was performed [region A, Figs. 2(b)2(d); region B, Fig. 3] (b) Time-gated autofluorescence microscopy image of region $\mathrm{A}$ at $\times 20$ magnification of the section, upon irradiation with a pulsed diode laser (35 ps, $405 \mathrm{~nm}, 40 \mathrm{MHz})$. (c) FLIM map obtained from a series of 21 images including (b), recorded at different delays with respect to the excitation pulse. A single-exponential decay was fitted, and the resulting fluorescence lifetime $\tau_{0}$ is represented in a false colour scale with the range indicated. Purple/blue colours represent fast fluorescence decays; yellow/red colors, slow decays. (d) Merged lifetime map formed by combining the lifetime (c) and intensity (b) images. This allows the functional lifetime information to be related to the anatomical intensity image without losing information from either parameter.

image intensification can also be varied. The quasi steady-state time-gated image produced at the phosphor screen (the decay time of the screen phosphorescence is much slower than the refresh rate of the image, which is equal to the laser repetition rate) is relayed to a CCD camera, and the images are recorded through the frame grabber of the personal computer controlling the experiment.

We have applied our compact FLIM microscope to the study of thin sections of rat tissue. Figure 2(a) shows a wide-field light transmission microscope image of a $15-\mu \mathrm{m}$-thick section of unstained rat ear. Figure 2(b) shows a time-gated autofluorescence intensity image of the section. The weak tissue autofluorescence induced by the laser light provides sufficient contrast to distinguish between the elastic cartilage, the blood vessel and the surrounding tissue by their different intensities. The fluorescence decay profiles are calculated for each image pixel from a series of such time-gated images, each recorded at a different delay time with respect to the laser excitation pulse. To produce Fig. 2(c), we recorded images at 21 temporal positions and fitted a single exponential decay function to each image pixel according to Eq. (1) and generated a FLIM map by plotting the fluorescence lifetimes $\tau_{0}$ obtained from the fits. It exhibits a good lifetime contrast between the different tissue components, distinguishing them by their chemical composition and environment rather than by their morphological structure. In particular, we find strong contrast between the elastic cartilage, other connective tissue, and the blood vessel-with differentiation between the vessel wall (media), the inner wall layer of the vessel (intima), and the clotted blood within the vessel. Since the fluorescence lifetime is chemically specific, it can be used to identify the different components by their value of $\tau_{0}$, which demonstrates the advantage of FLIM over conventional intensity-based microscopy. However, the FLIM map does not maintain the structural features of the sample, which can be seen only in the intensity image [Fig. 2(b)]. A solution to this problem is obtained by merging the lifetime and intensity images. This allows the functional lifetime information to be related to the anatomical intensity image without loss of information from either parameter. Since the false color scale of Fig. 2(c) is defined in the hue, saturation, and value (HSV) color space with the value being constant, the intensity image can be assigned to this value plane. The resulting merged lifetime map [Fig. 2(d)] clearly demonstrates the use of this representation, combining the structural tissue features with functional information in a single image.

For the calculation of the FLIM maps in Figs. 2(c) and 2(d), a single exponential decay of the fluorescence was assumed. As pointed out above, this assumption is not necessarily correct for heterogeneous biological tissue. To study this issue, we have compared the performance of different decay models, in particular the single-exponential [Eq. (1)], the double-exponential [Eq. (2)], and the stretchedexponential function [Eq. (3)]. Figure 3 shows the results of this comparison applied to FLIM data acquired of the magnified blood vessel [region B in Fig. 2(a)]. The time-gated autofluorescence image in Fig. 3(a) shows almost no structural details, apart from the demarcation between vessel tissue (high intensity) and vessel cavity with some clotted blood (very low intensity). In contrast, in the FLIM map, obtained by fitting of a single exponential function to the decay [Fig. 3(b)], the intima, and tissue surrounding the vessel (long lifetimes) are strongly contrasted from the medial layer of the wall (short lifetimes) and the vessel cavity (very short lifetimes). This is again a clear example of the power of FLIM to provide functional contrast that is not attainable with intensity measurements. We have applied the StrEF to fit the decay, and the mean lifetime $\langle\tau\rangle$ is plotted in Fig. 3(c). The contrast between different tissue constituents is at least as good as the one obtained with the single-exponential function, and in fact is slightly better at contrasting the vessel from the surrounding tissue. However, the crucial difference is that the StrEF describes the fluorescence decay better than the single exponential, as can be seen in Fig. 4, where the decay of a single pixel of the data is shown. This 


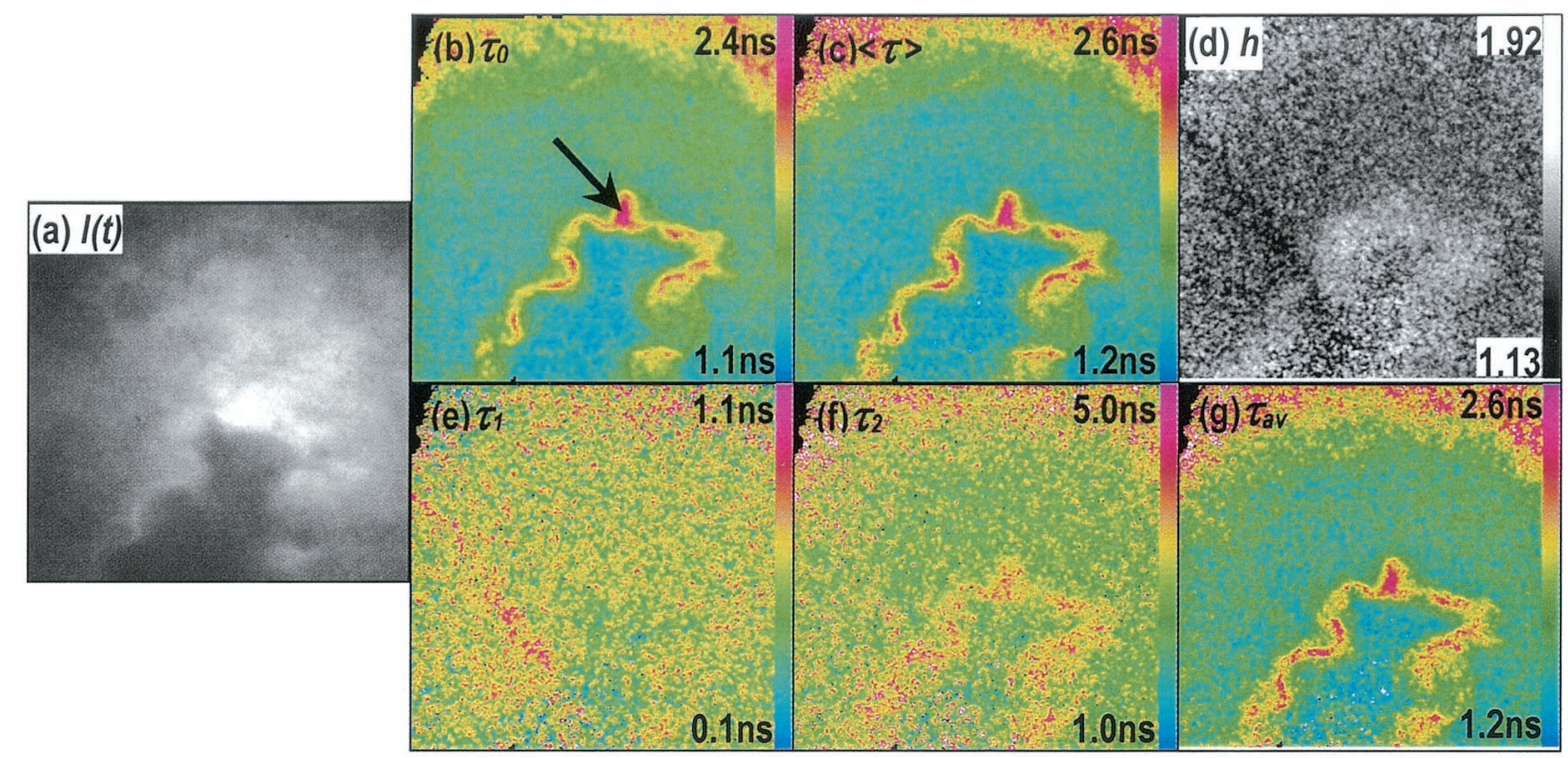

Fig. 3. Comparison of the performance of different decay models applied to FLIM microscopy data acquired from region B of the unstained tissue section at $\times 100$ magnification. (a) Time-gated fluorescence image (b) FLIM map obtained from a single exponential fit, displaying the lifetime $\tau_{0}$. [The arrow indicates the pixel used in (Fig. 4) to analyze the decay] (c) FLIM map obtained from a stretched exponential fit, displaying the mean lifetime $\langle\tau\rangle$. (d) Heterogeneity map obtained from a stretched exponential fit, displaying the heterogeneity parameter $h$. (e)-(g) FLIM maps obtained from a double exponential fit, displaying (e) the fast decay constant $\tau_{1}$, (f) the slow decay constant $\tau_{2}$, and (g) the (physically meaningless) weighted average lifetime $\tau_{\mathrm{av}}$.

confirms again that the StrEF is the more appropriate model to describe fluorescence decay in complex biological tissue, in agreement with our earlier findings.

A further important advantage of the StrEF is that additional contrast can be provided by exploiting the heterogeneity parameter $h$. Figure 3(d) shows a grayscale map of $h$, which is a direct measure of the width of the continuous lifetime distribution. Despite the noise, three different regions are well con-

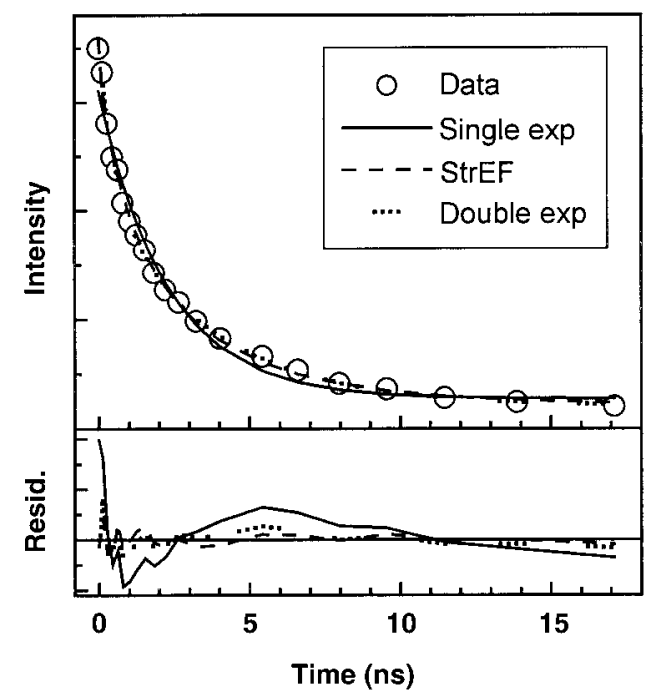

Fig. 4. Shows the sampled fluorescence intensity decay (circles) of a single pixel of the artery wall [arrow in Fig. 3(b)] comparing the performance of the fitting models (curves). The lower part of this graph shows the residuals of the fits. trasted: The relatively broad band of the inner vessel wall (very high $h$ values, corresponding to a broad lifetime distribution), which is spatially more homogeneous in the $h$ map than in the $\langle\tau\rangle$ or $\tau_{0}$ maps; a diagonal streak of unknown origin (very low $h$ values), which cannot be seen in either the $\langle\tau\rangle$ or $\tau_{0}$ map; and the vessel bulk (intermediate $h$ values). This additional information provided by the $h$ parameter is complementary to the lifetime information and has the potential to increase the specificity of tissue diagnosis.

When fitting to a double-exponential decay, we find that only by plotting the weighted average value $\tau_{\text {av }}$ [Fig. $3(\mathrm{~g})$ ] can we obtain a relatively good contrast and spatial image quality, ${ }^{27}$ although it is still notably noisier than either the single- or stretchedexponential function FLIM maps. The FLIM maps of the individual discrete components $\tau_{1}$ and $\tau_{2}$ [Figs. $3(e)$ and 3(f), respectively] exhibit very poor contrast and spatial image quality because of the reduced $\mathrm{S} / \mathrm{N}$ ratio, caused by the separation into two components. Interestingly, the features shown by the two components together represent to some extent the information given by the $h$ map [Fig. 3(d)]; i.e., the diagonal streak is seen in the map of $\tau_{1}$ and the broadband of the intima in the map of $\tau_{2}$. However, two maps are needed for this information, whereas a single map of the heterogeneity parameter contains both features. We also wish to stress that, although the maps of $\tau_{\mathrm{av}}$ from the double-exponential decay fit do provide reasonable contrast and image quality, this parameter is physically meaningless and therefore cannot be used for quantitative analysis. ${ }^{13}$ This is because the 
weighted average of two discrete components, which is the basis of the double-exponential model, would give a value somewhere in between these two components, which would be inconsistent with this discrete model. Furthermore, the double-exponential model assumes over the entire field of view the presence of either a single type of fluorophore with two discrete decay constants or two monoexponentially decaying fluorophores. This is a highly unrealistic assumption for a heterogeneous biological tissue. The fact that the double exponential model provides a similarly good fit as the StrEF is therefore solely due to the higher number of fitting parameters and not to its appropriateness as a fitting model for these data. In contrast, the StrEF does not require an arbitrary assumption of discrete components and allows the presence of a broad range of fluorescence lifetimes. The fitting calculation for the StrEF is also considerably faster than for the double exponential model. Thus the StrEF not only yields an excellent fit to the data (cf residues in Fig. 4) but it is also provides a better representation of the underlying biophysics.

\section{Determination of the Exponential Components by Means of Inverse Laplace Transformation}

We have investigated the possibility of extracting the actual exponential components from the decay profile data, in order to assess whether discrete components or a continuous distribution is more appropriate to describe fluorescence decays in biological tissue. Although the use of the StrEF does not require the determination of the distribution of lifetimes $\rho(\tau)$ [Eqs. (3) and (4) are alternative expressions], once determined, the first moment of this distribution corresponds to the mean lifetime $\langle\tau\rangle$ given in Eq. (5). Other moments of the distribution could be of interest as alternative plotting parameters, potentially providing additional contrast in FLIM maps as demonstrated by the heterogeneity parameter. A standard method to extract exponential components is using an inverse Laplace transformation. The conTin program written by Stephen Provencher is based on this method and has been successfully applied to many other fields of physics. ${ }^{28}$ We have begun to implement the corresponding algorithm in our analysis of fluorescence decay data. The algorithm has been tested on simulated single- and double-exponential decay data to ensure that the output of the algorithm does indeed correspond to one or two discrete components, respectively (results not shown). We then applied the algorithm to experimental decay data. Figure 5(a) shows the lifetime components extracted from the single pixel decay data in Fig. 4 by means of CONTIN. A very broad distribution, characteristic of a stretched exponential decay with a heterogeneity parameter $h>1$, can be observed, as opposed to one or two discrete components. Although more research is required for determining the exact shape and width of the distribution, this result supports our experimental finding that the decay data in biological tissue are better described by the stretched-exponential
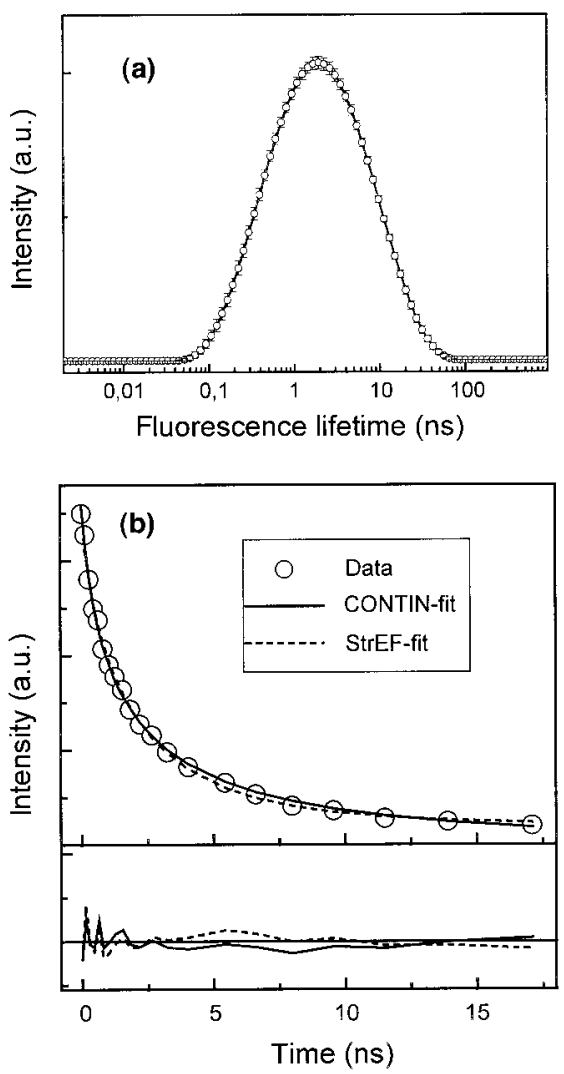

Fig. 5. (a) Fluorescence lifetime distribution as extracted by the CONTIN algorithm from experimental fluorescence decay data of a single pixel [marked by an arrow in Fig. 3(b)]. (b) Fluorescence decay data (circles) from this single pixel with fitted curves provided by the CONTIN algorithm and by the StrEF. The residuals of the fits, given in the lower part of the figure, show a comparable goodness of fit.

function than a multiexponential function. Essentially we believe that it is safer and more useful to fit the unknown continuous lifetime distribution expected for biological tissue to a generic continuous distribution model rather than to a discrete model.

The fitted StrEF that was used to produce the FLIM map in Fig. 3(c) and the heterogeneity map in Fig. 3(d) overlaps well with the fitted curve provided by CONTIN, which uses the extracted components of its output to fit the data [Fig. 5(b)]. The small differences between the two curves are due to the intermediate step in the Laplace transformation in the CONTIN algorithm. It is worth noting that the algorithm yields a number of possible solutions of the transformation and it is critically important to select the most appropriate one. CONTIN uses parsimony to select the best solution, displaying the least amount of information that was not already known by attempting to minimize the degrees of freedom. Nevertheless, the other solutions are also accessible to the user and their performance in fitting the data, to confirm that the parsimony criterion is appropriate for a specific case. In the case of the single pixel decay data shown in Fig. 5, all other solutions did have significantly higher residuals in the fit of the decay data 


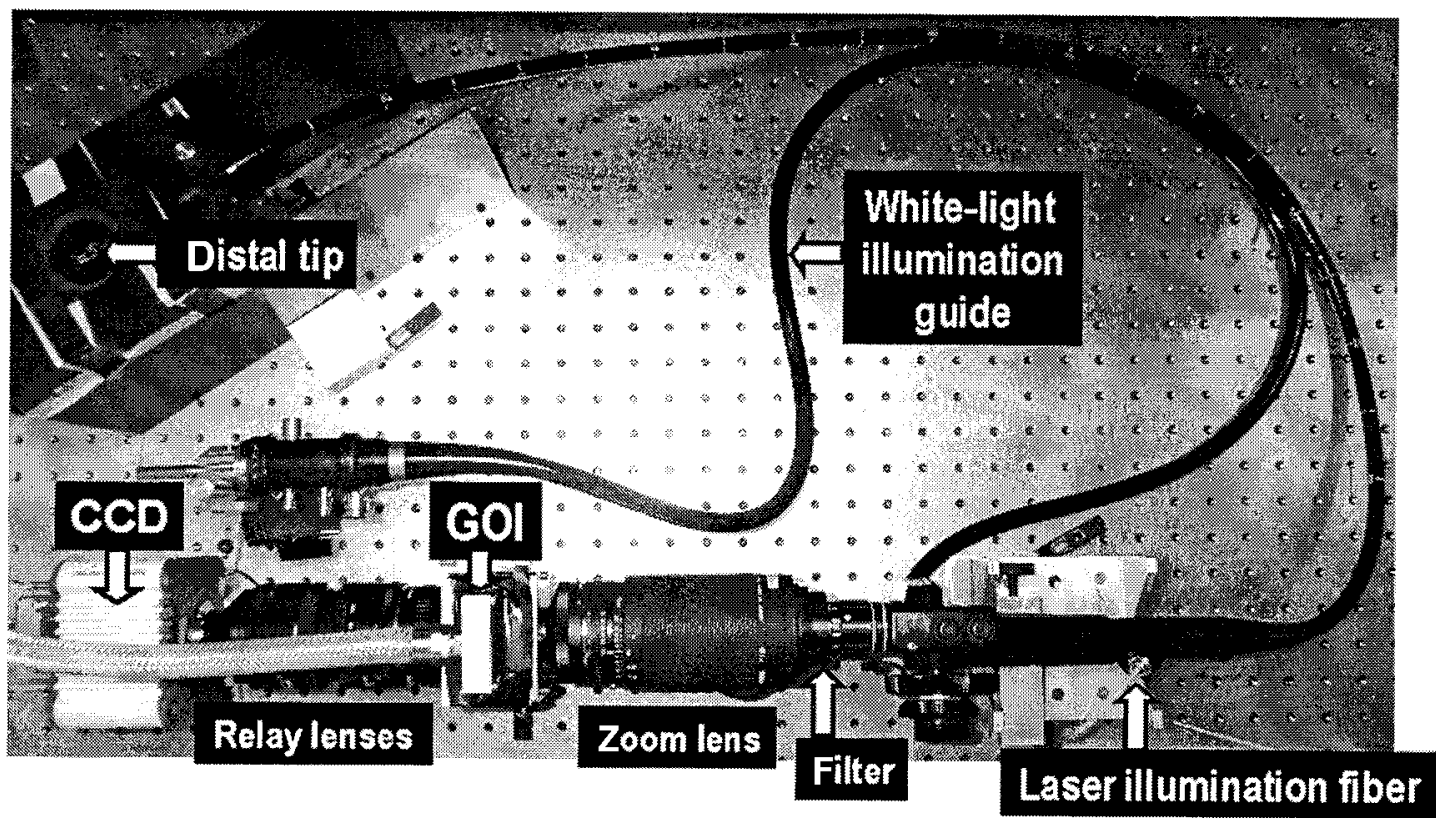

Fig. 6. Experimental setup for FLIM endoscopy.

and/or the lifetime distribution. In this respect, the results given by CONTIN can be considered an independent verification of our previous finding that the autofluorescence in biological tissue displays a broad distribution of fluorescence lifetimes, even for a single pixel, and that the decay can be well described by a stretched exponential function. To determine the shape and width of the lifetime distribution with high precision, we are currently working on a detailed study of the inverse Laplace method, systematically determining all factors that might influence the extracted lifetime distribution, such as the number of time gates acquired, sampling range, temporal resolution of the system and $\mathrm{S} / \mathrm{N}$ ratio.

\section{Fluorescence Lifetime Imaging Endoscopy}

Although the potential of this FLIM microscope when combined with the appropriate decay model in the form of the StrEF is evident for functional in vitro studies of biological tissue sections, in vivo studies require a more flexible experimental apparatus. To address this issue, we have begun to develop a FLIM endoscope, for which the experimental setup can be seen in Fig. 6. A commercially available flexible endoscope was used (FiberDuodenoscope JF-1T20, Olympus), from which the output of the imaging fiber bundle was coupled by a camera lens (Nikon standard lens, $f=50 \mathrm{~mm}, 1.4$, or Sigma zoom lens $f=$ 70-200 mm, 3.5-4.5) to the GOI. As with the FLIM microscope, the time-gated images at the phosphor screen of the GOI were relayed by a system of two camera lenses (Nikon $f=50 \mathrm{~mm}, 1.4$ ) front-tofront onto a CCD camera. A long-pass filter between the endoscope and the GOI lens blocked residual excitation light. Excitation was provided by a frequency-doubled femtosecond oscillator (Tsunami, Spectra-Physics) producing pulses of $100 \mathrm{fs}$ at $415 \mathrm{~nm}$ and $80-\mathrm{MHz}$ repetition rate with a maximum average power of $30 \mathrm{~mW}$. Fluorescence excitation of the sample was performed by means of coupling the laser light into a light guide (diameter, $1 \mathrm{~mm}$ ), fed through the biopsy port of the endoscope. The low transmission efficiency of the light guide (50\%), combined with the low collection angle of the endoscope and the relatively large field of view to excite (typically $3 \mathrm{~cm} \times 3 \mathrm{~cm}$ ), made the use of tens of milliwatts of excitation power necessary. The white-light illumination channel of the endoscope was preserved, and white-light reflection images were acquired by use of the dc mode of the GOI.

The temporal dispersion of the system has been characterized by use of a streak camera to measure the temporal width of the excitation pulses (100 fs) after passage through the light guide and then the imaging fiber bundle. The temporal broadening of the pulses during passage through the standard multimode imaging fiber bundle was close to the resolution of the streak camera $(>35 \mathrm{ps})$. The transmitted pulse duration was measured to be $\sim 46 \mathrm{ps}$ (without deconvolution) [Fig. 7(a)], which implies that the effect of the temporal broadening on the measured fluorescence lifetimes (with typical values between hundreds of picoseconds to several nanoseconds) is very small. As with the imaging bundle, little temporal broadening was observed when the laser was coupled perpendicular to the flat light guide end, whereas an appreciable broadening was found for coupling in at an angle. The advantage of angled coupling is that the spatial illumination becomes more homogeneous as the angle increases until, eventually, the entire field (limited by the NA of the fiber) is homogeneously illuminated. Therefore a compromise between temporal broadening and homogeneous illumination had to be found. We kept the 

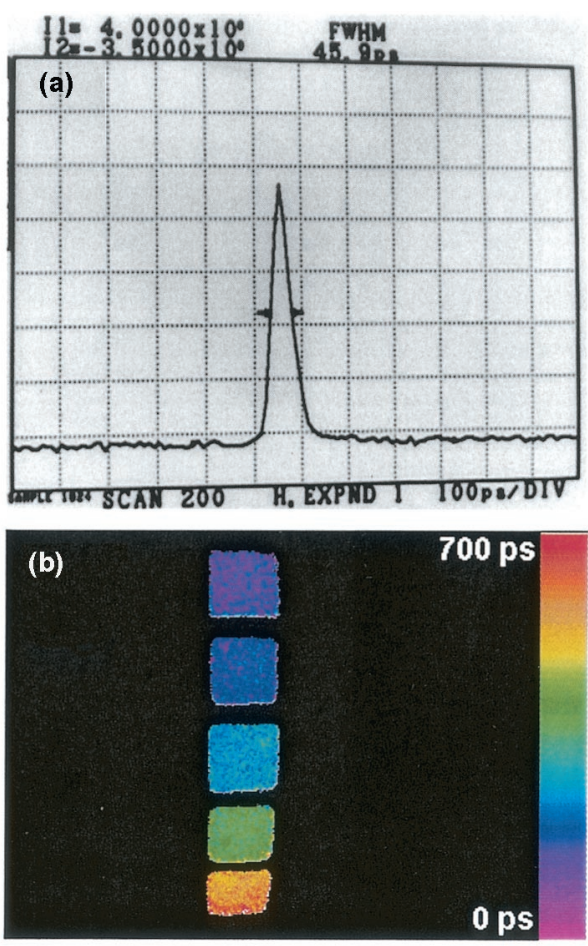

Fig. 7. (a) Temporal broadening of $100-\mathrm{fs}$ pulses to $46 \mathrm{ps}$ in the multimode imaging bundle of the endoscope, as measured with a Streak Camera (resolution, $\sim 35 \mathrm{ps}$ ). (b) FLIM endoscopy images of a multiwell plate sample containing the dye DASPI in solvents of different viscosity (ethanol/glycerol ratios from bottom to top: $20 / 80,40 / 60,60 / 40,80 / 20,100 / 0)$. As the viscosity increases from top to bottom, the lifetime increases. The false color scale spans from $0 \mathrm{ps}$ (purple) to $700 \mathrm{ps}$ (red).

total temporal dispersion experienced in the light guide and imaging fibers close to the temporal resolution of our FLIM system. In a microscopic configuration, the system can resolve lifetime differences as short as tens of picoseconds ${ }^{6}$ but requires a relatively strong fluorescent signal because of the low gain at the shortest gate width $(100 \mathrm{ps})$. The weak autofluorescence in the tissue and small detection angle of the endoscope required a larger gate width $(400 \mathrm{ps})$ to be used, which has a considerably higher gain. It is worth noting, though, that the large gate width does not limit the temporal resolution to $400 \mathrm{ps}$, because the temporal shape of the gate pulse is approximately rectangular. Thus only the apparent fluorescence rise time is affected whereas the decay time is unchanged. A method to quantify the effective temporal resolution of the FLIM endoscope is to perform a measurement of a multiwell plate sample of the dye DASPI in solvents of different viscosities. ${ }^{26,29}$ DASPI has a very short fluorescence lifetime as a result of nonradiative relaxation occurring through internal twisting. The fluorescence lifetime can be increased by addition of a high-viscosity solvent, which slows down the twisting motion. Figure 7(b) shows this effect clearly; i.e., the lifetime increases gradually with viscosity, which demonstrates the correct operation of our FLIM endoscope. The shortest
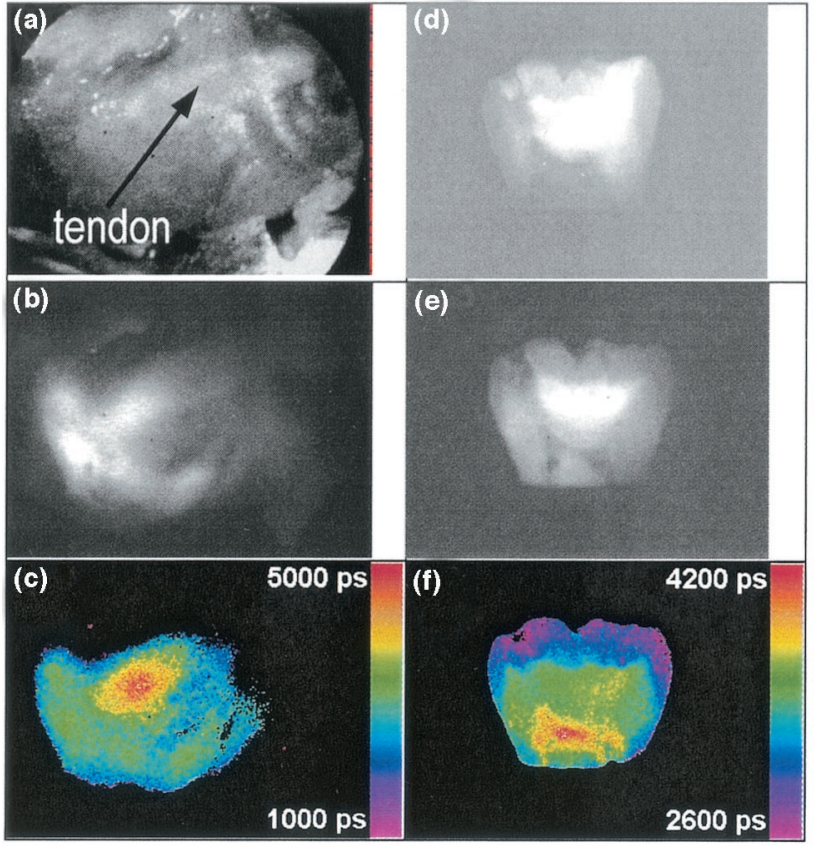

Fig. 8. Endoscopy images of a rabbit joint (a)-(c) and a longitudinal section of a human tooth (d)-(f): (a) and (d) dc white-light reflection images, (b) and (e) time-gated fluorescence intensity images (c) and (f) FLIM maps, contrasting the different regions according to their characteristic fluorescence lifetime. Rabbit joint (c): The region where the tendon attaches to the bone exhibits long lifetimes (red/yellow). Human tooth (f): The outer enamel layer has a much shorter lifetime (violet) than the dentine (green) or the root canal region (yellow/red).

measured lifetime in this map corresponding to DASPI in ethanol is $150 \mathrm{ps}$, which compares with an expected value of $\sim 40 \mathrm{ps}^{29}$ Therefore $150 \mathrm{ps}$ can be considered the effective limit for the lifetime resolution of our FLIM endoscope.

Having demonstrated that our FLIM endoscope is able to contrast the fluorescence lifetime of dye solutions, we have begun to investigate its applications to biological tissue. Figure 8(a) shows a white-light image of a previously frozen rabbit knee joint region. The joint capsule was opened to reveal a tendon attached to the bone, which is surrounded by synovial and other tissue. The time-gated fluorescence intensity image [Fig. 8(b)] highlights both the tendon and the area where it is attached. The corresponding FLIM map [Fig. 8(c)] shows clear functional contrast between the region where the tendon attaches to the bone and the surrounding tissue.

We have also applied this FLIM endoscope to the study of an extracted human tooth. Figure 8(d) shows a white-light image of a longitudinal section of a tooth. The different reflectivities/specular reflections make it difficult to identify the different regions. The time-gated fluorescence intensity image [Fig. $8(\mathrm{e})$ provides some contrast between the different regions, but variations in the excitation intensity and fluorophore concentration across the sample make identification difficult. The FLIM map [Fig. 8(f)] is, 
however, not affected by the intensity variations and clearly contrasts the enamel, dentin, and root canal regions by their characteristic fluorescence lifetimes. This improved demarcation illustrates the potential utility of fluorescence lifetime imaging in endoscopic studies. It is interesting to note that the tooth, which has caries in it at the junction between the enamel and dentine, is not showing any signal from the decayed area but shows excellent discrimination of the enamel and dentine. Further developments in mapping the FLIM signature of these features may lead to their discrimination.

We note that the FLIM maps in Figs. 8(c) and 8(f) were derived by use of a single exponential fit, rather than a StrEF fit. This was necessary because of the relatively high noise present in these decay data sets, which effects the StrEF fit more significantly than the single-exponential fit. As we have shown in Section 3 , the use of the single exponential is a reasonable approximation for biological tissue because it maintains the relative lifetime contrast between different tissue constituents and provides a good image quality. Nevertheless, the goodness of fit is inferior to what may be obtained with a StrEF and, more importantly, it does not yield additional information in the form of a heterogeneity parameter. We are confident though that a custom-designed endoscope with optimized illumination and detection efficiencies will provide a $\mathrm{S} / \mathrm{N}$ ratio comparable with that obtained with the FLIM microscope, which would then allow the use of the StrEF also for FLIM endoscopy.

The acquisition time for FLIM endoscopy is still too long to meet clinical requirements that need an update time near real-time. For instance, the data set upon which the FLIM map in Fig. 8(f) is based took five minutes to acquire. The subsequent processing to produce the FLIM map took less than $10 \mathrm{~s}$. However, a custom-designed endoscope would reduce considerably the acquisition time. The acquisition and processing can be speeded up further by reduction of the number of time gates, which is particularly effective when the decay characteristics of the features under investigation are known (from high-precision, single-point measurements) so that a lifetime contrast between the different tissue types or states of tissue can be achieved with very few time gates. In FLIM microscopy we have already demonstrated the performance of near real-time FLIM with an update time of only $3 \mathrm{~s}$, including acquisition and processing on a personal computer (Cyrix $\mathrm{P} 200+$ processor). We believe that a FLIM update rate of several lifetime maps/s is feasible with the latest personal computers.

\section{Conclusion}

We have successfully applied fluorescence lifetime imaging (FLIM) to the study of biological tissue, exploiting autofluorescence of tissue constituents upon excitation with laser light. The characteristic fluorescence properties of different endogenous tissue fluorophores, in particular their characteristic fluo- rescence lifetimes, yield functional contrast between different types or states of tissue as opposed to intensity-based contrast, which is affected by the fluorophore concentration. Because of the complex nature of tissue, which comprises a range of microenvironments and protein conformations, a continuous distribution of fluorescence lifetimes, as opposed to discrete lifetime components, is expected to be present within a single image pixel. The observed fluorescence decays are therefore best described by the stretched exponential function (StrEF), owing to its mathematical correspondence with a continuous lifetime distribution. We confirmed this hypothesis through two independent studies. First, by comparing the performance of different decay models, we find that the StrEF provides excellent fits to the data, requiring a considerably shorter calculation time than a multiexponential model and generally providing significantly better FLIM maps. Second, by extracting the actual exponential decay components using an inverse Laplace transformation, we obtain a continuous distribution as opposed to discrete components. We note that the StrEF also provides an additional contrast parameter: heterogeneity, which is a measure of the width of the lifetime distribution.

We believe that our FLIM microscope in combination with the StrEF analysis is an appropriate and useful tool for the clinical study of tissue sections. The use of a compact picosecond diode laser as the excitation source permits a small footprint (of $\sim 0.25$ $\mathrm{m}^{2}$ ) for the whole system. The results obtained with our FLIM endoscope, although they successfully demonstrate the viability of this functional imaging modality, show the need for further optimization. Although temporal dispersion in the fiber endoscope is shown not to be a limiting factor and correct functional contrast is achieved in the samples studied, the observed low detection efficiency combined with nonoptimized illumination conditions led to significant noise in the fluorescence lifetime maps. This prevented us from using the appropriate stretched exponential function but we note that the singleexponential fit to complex fluorescence decay data still provides useful contrast for clinical applications. We are confident that a custom-designed endoscope with optimized illumination and detection efficiencies will provide a $\mathrm{S} / \mathrm{N}$ ratio comparable with that obtained with the FLIM microscope. Such a customdesigned endoscope would also reduce considerably the acquisition time, which is currently beyond the tolerable level for clinical applications.

Funding for this research from the UK Engineering and Physical Sciences Research Council (EPSRC) and the Biotechnology and Biological Sciences Research Council (BBSRC) is gratefully acknowledged. D. S. Elson acknowledges an EPSRC Cooperative Awards in Science and Engineering (CASE) studentship with Kentech Instruments Ltd. S. E. D. Webb acknowledges an EPSRC studentship. 


\section{References and Notes}

1. G. A. Wagnieres, W. M. Star, and B. C. Wilson, "In vivo fluorescence spectroscopy and imaging for oncological applications," Photochem. Photobiol. 68, 603-632 (1998).

2. N. Lange, P. Jichlinski, M. Zellweger, M. Forrer, A. Marti, L. Guillou, P. Kucera, G. Wagnieres, and H. van den Bergh, "Photodetection of early human bladder cancer based on the fluorescence of 5-aminolaevulinic acid hexylester-induced protoporphyrin IX: a pilot study,” Br. J. Cancer 80, 185-193 (1999).

3. R. Ackroyd, C. Kelty, N. Brown, and M. Reed, "The history of photodetection and photodynamic therapy," Photochem. Photobiol. 74, 656-669 (2001).

4. R. Y. Tsien and M. Poenie, "Fluorescence ratio imaging: a new window into intracellular ionic signalling," Trends Biochem. Sci. 11, 450-455 (1986).

5. M. Sinaasappel and H. J. C. M. Sterenborg, "Quantification of the hematoporphyrin derivative by fluorescence measurement using dual-wavelength excitation and dual-wavelength detection," Appl. Opt. 32, 541-548 (1993).

6. K. Dowling, M. J. Dayel, M. J. Lever, P. M. W. French, J. D. Hares, and A. K. L. Dymoke-Bradshaw, "Fluorescence lifetime imaging with picosecond resolution for biomedical applications," Opt. Lett. 23, 810-812 (1998).

7. P. I. H. Bastiaens and A. Squire, "Fluorescence lifetime imaging microscopy: spatial resolution of biochemical processes in the cell," Trends Cell Biol. 9, 48-52 (1999).

8. R. Cubeddu, A. Pifferi, P. Taroni, A. Torricelli, G. Valentini, F. Rinaldi, and E. Sorbellini, "Fluorescence lifetime imaging: an application to the detection of skin tumors," IEEE J. Sel. Top. Quantum Electron. 5, 923-929 (1999).

9. W. Becker, A. Bergmann, and G. Weiss, "Lifetime imaging with the Zeiss LSM-510," in Multiphoton Microscopy in the Biomedical Sciences II, A. Periasamy, W. M. Keck, and P. T. C. So, eds., Proc. SPIE 4620, 30-35 (2002).

10. See, for instance, P. C. Schneider and R. M. Clegg, "Rapid acquisition analysis and display of fluorescence lifetimeresolved images for real-time applications," Rev. Sci. Instrum. 68, 4107-4119 (1997).

11. See, for instance, G. Valentini, C. D'Andrea, D. Comelli, A. Pifferi, P. Taroni, A. Torricelli, R. Cubbeddu, C. Battaglia, C. Consolandi, G. Salani, L. Rossi-Bernardi, and G. De Bellis, "Time-resolved DNA-microarray reading by an intensified CCD for ultimate sensitivity," Opt. Lett. 25, 1648-1650 (2000).

12. J. R. Lakowicz, Principles of Fluorescence Spectroscopy (Kluwer, New York, 1999).

13. K. C. Benny Lee, J. Siegel, S. E. D. Webb, S. Lévêque-Fort, M. J. Cole, R. Jones, K. Dowling, M. J. Lever, and P. M. W. French, "Application of the stretched exponential function to fluorescence lifetime imaging," Biophys. J. 81, 1265-1274 (2001).

14. H. Ina, H. Shibuya, I. Ohashi, and M. Kitagawa, "The frequency of a concomitant early esophageal cancer in male patients with oral and oropharyngeal cancer. Screening results using Lugol dye endoscopy," Cancer 73, 2038-2041 (1994).
15. J. Y. Qu, J. W. Hua, and Z. J. Huang, "Correction of geometrical effects on fluorescence imaging of tissue," Opt. Commun. 176, 319-326 (2000).

16. J. Y. Qu, "Real time calibrated fluorescence imaging of tissue in vivo by using the combination of fluorescence and crosspolarized reflection," in Biomedical Topical Meetings, Vol. 71 of OSA Trends in Optics and Photonics Series (Optical Society of America, Washington, D.C., 2002), pp. 485-487.

17. H. Zeng, A. Weiss, R. Cline, and C. E. MacAuley, "Real-time endoscopic fluorescence imaging for early cancer detection in the gastrointestinal tract," Bioimaging 6, 151-165 (1998).

18. T. McKechnie, A. Jahan, I. Tait, A. Cuschieri, W. Sibbett, and M. Padgett, "An endoscopic system for the early detection of cancers of the gastrointestinal tract," Rev. Sci. Instrum. 69, 2521-2523 (1998).

19. Y. S. Sabharwal, A. R. Rouse, L. Donaldson, M. F. Hopkins, and A. F. Gmitro, "Slit-scanning confocal microendoscope for high-resolution in vivo imaging," Appl. Opt. 38, 7133-7144 (1999).

20. G. J. Tearney, R. H. Webb, and B. E. Bouma, "Spectrally encoded confocal microscopy," Opt. Lett. 23, 1152-1154 (1998).

21. J. Mizeret, G. Wagnieres, T. Stepinac, and H. Van Den Bergh, "Endoscopic tissue characterization by frequency-domain fluorescence lifetime imaging (FD-FLIM)," Las. Med. Sci. 12, 209-217 (1997).

22. D. R. James and W. R. Ware, "A fallacy in the interpretation of fluorescence decay parameters," Chem. Phys. Lett. 120, 455459 (1985).

23. J. R. Alcala, E. Gratton, and F. G. Prendergast, "Fluorescence lifetime distributions in proteins," Biophys. J. 51, 597-604 (1987).

24. J. R. Alcala, "The effect of harmonic conformational trajectories on protein fluorescence and lifetime distributions," J. Chem. Phys. 101, 4578-4584 (1994).

25. F. Alvarez, A. Alegría, and J. Colmenero, "Relationship between the time-domain Kohlrausch-Williams-Watts and frequency-domain Havriliak-Negami relaxation functions," Phys. Rev. B 44, 7306-7312 (1991).

26. D. S. Elson, J. Siegel, S. E. D. Webb, S. Lévêque-Fort, M. J. Lever, P. M. W. French, K. Lauritsen, M. Wahl, and R. Erdmann, "Fluorescence lifetime system for microscopy and multiwell plate imaging with a blue picosecond diode laser," Opt. Lett. 27, 1409-1411 (2002).

27. Spatial image quality is a measure of lifetime variability between connected pixels within regions of the same average lifetime; image contrast measures lifetime variation between averaged regions.

28. S. W. Provencher, "Contin: a general purpose constrained regularization program for inverting noisy linear algebraic and integral equations," Comput. Phys. Commun. 27, 229-242 (1982).

29. K. Dowling, M. J. Dayel, S. C. W. Hyde, P. M. W. French, M. J. Lever, J. D. Hares, and A. K. L. Dymoke-Bradshaw, "High resolution time-domain fluorescence lifetime imaging for biomedical applications,” J. Mod. Opt. 46(2), 199-209 (1999). 